Draft Version August 7, 2018

Preprint typeset using LTEX style emulateapj v. 2/16/10

\title{
NO EVIDENCE OF OBSCURED, ACCRETING BLACK HOLES IN MOST Z=6 STAR-FORMING GALAXIES
}

\author{
CHRIS J. WILLOTT ${ }^{1}$, \\ Draft version August 7, 2018
}

\begin{abstract}
It has been claimed that there is a large population of obscured, accreting black holes at high-redshift and that the integrated black hole density at $z=6$ as inferred from X-ray observations is $\sim 100$ times greater than inferred from optical quasars. I have performed a stacking analysis of very deep Chandra X-ray data at the positions of photometrically-selected $z=6$ galaxy candidates. It is found that there is no evidence for a stacked X-ray signal in either the soft (0.5-2 keV) or hard (2-8 keV) X-ray bands. Previous work which reported a significant signal is affected by an incorrect method of background subtraction which underestimates the true background within the target aperture. The puzzle remains of why the $z=6$ black hole mass function has such a flat slope and a low normalization compared to the stellar mass function.
\end{abstract}

Subject headings: cosmology: observations — galaxies: active — galaxies: high-redshift — X-rays: galaxies

\section{INTRODUCTION}

The intimate relationship between galaxies and their nuclear black holes is a key, but as yet unsolved, issue in astrophysics. Whilst there is a tight correlation between stellar velocity dispersion and black hole mass in the local universe (Ferrarese \& Merritt 2000; Gebhardt et al. 2000), it is still unknown how this relationship evolves at higher redshifts when most star formation, galaxy assembly and black hole accretion occurred. Determining the evolution will constrain which of the many theoretical explanations for the relationship are responsible.

Willott et al. (2010) showed that the $z=6$ black hole mass function based on optical quasars is substantially lower than expected based on the $z=6$ stellar mass function (Stark et al. 2009) if the local correlation between black hole and galaxy mass does not evolve at high-redshift. On the other hand, the masses of black holes in luminous $z=6$ quasars appear to be higher than locally for a given galaxy mass (Wang et al. 2010), although these measurements are potentially influenced by the selection bias discussed by Lauer et al. (2007). These observations could be explained by a steeper highredshift slope to the black hole - galaxy mass relation or a mass-dependent active black hole duty cycle (Volonteri \& Stark 2011; Fiore et al. 2011a).

Fiore et al. (2011a) searched for X-ray emission at the locations of all galaxies with photometric redshifts $z>5.8$ in the Chandra Deep Field South (CDF-S). They found significant X-ray emission in only two galaxies of which at least one has a plausible lower $z$ photometric solution, enabling them to place an upper limit on the space density of low-luminosity active galaxies at $z=6$. Treister et al. (2011; hereafter T11) found a significant signal in the stacked X-ray flux at the locations of $197 z \approx 6$ Lyman break galaxies (LBG). The data in each X-ray band was stacked independently and they found a $5 \sigma$ detection in the soft $(0.5-2 \mathrm{keV})$ band and a $6.8 \sigma$ detection in the hard $(2-8 \mathrm{keV})$ band. This corresponds to typical $\mathrm{X}$-ray luminosities of $z=6$ galaxies of $9.2 \times 10^{41} \mathrm{erg} \mathrm{s}^{-1}$ in the soft band and $8.4 \times 10^{42} \mathrm{erg} \mathrm{s}^{-1}$ in the hard band. The high ratio of these luminosities indicates a hard X-ray spectrum which, considering the sizeable $k$-correction at $z \approx 6$

\footnotetext{
${ }^{1}$ Herzberg Institute of Astrophysics, National Research Council, 5071 West Saanich Rd, Victoria, BC V9E 2E7, Canada; chris.willott@nrc.ca
}

(soft band is rest-frame $3.5-14 \mathrm{keV}$ and hard band is restframe $14-56 \mathrm{keV}$ ), implies a typical obscuring column of $N_{H}>1.6 \times 10^{24} \mathrm{~cm}^{-2}$.

This X-ray emission from $z=6$ active galactic nuclei can be used to infer an integrated black hole mass density at that epoch, making some assumptions about the population evolution, bolometric correction and radiative efficiency (see T11). This results in a factor of $\approx 100$ more black hole growth at high-redshift than had previously been inferred from the optical quasar luminosity function. The inference is that the black hole mass function is steeper than previously thought and dominated by low-mass, heavily obscured black holes. This steeper function would be more in line with theoretical expectations (Volonteri \& Stark 2011). However, the ratio of obscured to unobscured black holes would be more than an order of magnitude larger at $z=6$ than the values of $2-4 \mathrm{ob}$ served at $2<z<5$ (Treister et al. 2009; Fiore et al. 2011a).

None of the $197 z=6$ galaxies were individually detected at high significance in either band of the X-ray data (Alexander et al. 2003; Xue et al. 2011). The relatively high significance of the stacked signal combined with the number of objects being stacked indicates a significant contribution from many of the galaxies (T11 estimate $>30 \%$ ) or else some of them would have been detected individually. With none detected individually, but a great many lurking just below the flux detection threshold, it implies a very steep flux (luminosity) distribution which is surprising given the large range of galaxy luminosity and the expected range in black hole mass, accretion rate relative to Eddington, obscuring column, etc.

In order to better understand the origin of the stacked X-ray signal and implications for black hole mass evolution, I have analyzed the X-ray properties of photometrically-selected $z=$ 6 LBGs. In this Letter I describe my analysis and relate it to previous work. After submission of this Letter, Fiore et al. (2011b) published an independent analysis of the stacked signal from $z=6$ LBGs in the CDF-S. Those results will be discussed in Section 3.

\section{STACKING METHOD}

Many of the details of the method follow closely those described in the main paper and supplementary information of T11 and the interested reader is referred there for details. Two slightly different methods are used, one to produce the image 
stack for display purposes and one to optimally determine the stacked signal and its noise, hereafter the S/N stack. All the stacking is performed using the mean, rather than the median that is usually considered the more robust estimator (White et al. 2007), due to the fact we are dealing with low integer counts.

The list of $z=6$ LBGs used by T11 contains 151 galaxies in the CDF-S and 46 in the Chandra Deep Field North (CDF$\mathrm{N})$. Since the CDF-S $4 \mathrm{Ms}$ Chandra data is deeper than the CDF-N 2 Ms data, it contributes most of the weight and only the CDF-S field is considered here. The CDF-S 4 Ms Chandra images and catalogs (Xue et al. 2011) were downloaded from the public website. The sample of $151 \mathrm{CDF}-\mathrm{S}$ galaxies used was drawn from a larger pool of 355 unique CDF-S galaxies in Bouwens et al. (2006). As in T11, galaxies are not included if they have Chandra off-axis angles $>9$ arcmin or they have a neighboring X-ray source within $22 \operatorname{arcsec}$ in the 2 Ms Chandra catalog of Luo et al. (2008).

Due to the large variation in the Chandra point spread function (PSF) as a function of off-axis angle, an optimal photometric aperture extraction radius, $r$, is determined for each object position. Values of $r$ range from 1 arcsec close to the aim-point to 7 arcsec at $\approx 9$ arcmin off-axis. The background per pixel, determined as described below, is subtracted. The effective exposure varies somewhat across the field-of-view, so the net counts per pixel were divided by the exposure time to get a count rate per Ms. Because the targets with large offaxis angles have larger photometry apertures, they will contain a greater background signal and hence have higher noise. Therefore each target has a weighting factor, $w_{i}$, determined by the inverse noise squared, $n_{i}^{-2}$. Weighting factors are used for image stacks and S/N stacks. The total exposure time for the 151 sources is $5.4 \times 10^{8} \mathrm{~s}$, equivalent to $\approx 17$ years.

For the image stacks the weighted, background-subtracted count rate images are averaged. Images are kept in the native 0.492 arcsec pixels. There is no stretch of the images to correct for the varying PSF. For the S/N stacks the signal and noise for each target are calculated individually. The average stacked signal, $S$, is determined from the individual target signals, $s_{i}$, by $S=\sum_{i} w_{i} s_{i} / \sum_{i} w_{i}$ and the related noise is $N=\left(\sum_{i} w_{i}^{2} n_{i}^{2}\right)^{0.5} / \sum_{i} w_{i}$ (see appendix A of T11).

The final detail of the method and the one that is most important to this analysis is the estimation of the background count rate. The Chandra ACIS background is extremely low and even in the $4 \mathrm{Ms}$ data, most pixels have zero counts in each of the soft and hard bands (Xue et al. 2011). Nevertheless, the background is the largest source of uncertainty in the stacking analysis due to the very low signal. The background is a mixture of instrumental effects, diffuse galactic/extragalactic emission and unresolved extragalactic point sources (Markevitch et al. 2003).

Background counts are determined from the pixel values measured within an annulus from $2 r$ to 22 arcsec around each target position. Three methods of background subtraction are considered.

a - The background is determined from the mean value, $\mu$, of pixels within the background annulus.

b - The standard deviation, $\sigma$, and mean of all pixels within a radius of 22 arcsec around each target position is calculated. Any pixel within this circle with a

\footnotetext{
${ }^{3}$ http://www2.astro.psu.edu/ niel/cdfs/cdfs-chandra.html
}

value greater than $\mu+3 \sigma$ has its value set to $\mu$. For the rare cases where $\mu+3 \sigma<1$, only pixels with 2 or more counts were clipped. This method suppresses noise in the background annulus, but will also remove real signal from the stacked position.

c - The standard deviation and mean of all pixels within the background annulus from $2 r$ to 22 arcsec is calculated. Pixels in the background annulus with value greater than $\mu+3 \sigma$ had their values set to $\mu$. Again, for rare cases where $\mu+3 \sigma<1$, only pixels with 2 or more counts were clipped. This is the background method adopted by T11. This method has the seemingly attractive behaviour of eliminating noise and undetected point sources in the background determination whilst not eliminating flux from the target position. However, it will be shown that this leads to a considerable bias in a stacking analysis.

\section{RESULTS}

Image stacks using the three different background determination methods are shown in Fig. 1. Each image displays a 30 arcsec region and has been smoothed by a 7 pixel Gaussian. Methods (a) and (b) show no sign of an excess of flux at the center for either band. Method (c) shows significant positive signal in both bands.

Values for the stacked S/N for each method and band are given in Table 1 . These confirm that the only significant signal is for method (c). Indeed, the soft band for method (a) shows a negative flux, although it is only significant at $\mathrm{S} / \mathrm{N}=-1.3$ and hence not particularly unlikely to occur by chance. The values of signal and background for method (c) have been compared to those for the same targets in T11 and are very similar (Treister, priv. comm.). The stacked $\mathrm{S} / \mathrm{N}$ for method (c) of 4.7 for the soft band and 6.1 for the hard band are comparable, but slightly lower, than the results for the full sample (including CDF-N) of 197 sources which have S/N of 5 for soft and 6.8 for hard in $\mathrm{T} 11$.

There is an obvious reason why the stacked signal only appears for method (c). In this case, the background annulus has had $>3 \sigma$ pixels clipped, but the same clipping has not been applied to the target aperture. Whilst for each target this only makes a small difference to the background level $(\approx 10 \%)$, it is a systematic effect that builds up in the stack. $\sigma$ clipping makes sense for some data with sharp noise spikes, e.g. optical data affected by cosmic rays, but if it is to be applied then it must also be applied to the target positions to avoid a cumulative positive bias (as in method b), and it must be known that this will not remove signal from the sources being stacked. In the CDF-S Chandra case, where much of the background is due to faint sources below the detection threshold, it makes more sense to do no $\sigma$ clipping at all, i.e. method (a), and to leave the faint sources in both the target and background data, knowing that on average they will cancel out. Therefore, the optimum results are those of method (a) with measured $\mathrm{S} / \mathrm{N}$ of -1.3 and 0.1 in the soft and hard bands, respectively. These correspond to $3 \sigma$ limits on the count rate per galaxy of $<2.4 \times 10^{-7} \mathrm{ct} \mathrm{s}^{-1}$ and $<4.2 \times 10^{-7} \mathrm{ct} \mathrm{s}^{-1}$ in the soft and hard bands, respectively, where a correction has been applied for counts outside the photometric apertures. This hard band $3 \sigma$ upper limit is only half the reported hard band signal of T11.

Fiore et al. (2011b) also performed stacking of the CDF$S$ Chandra data at the known positions of $z=6$ LBGs from 


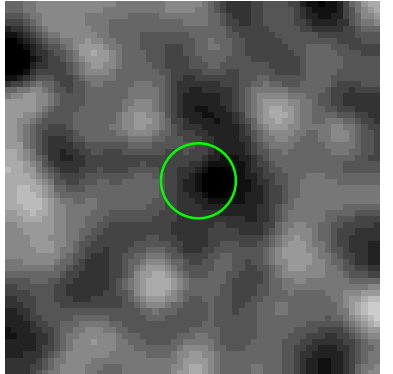

Soft Band

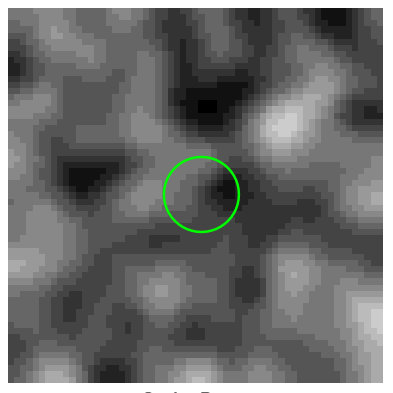

Soft Band

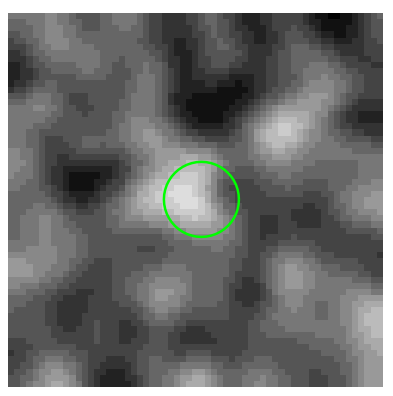

Soft Band

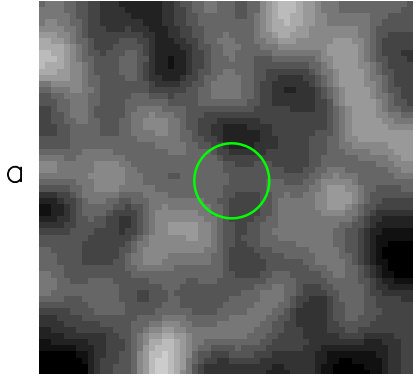

Hard Band

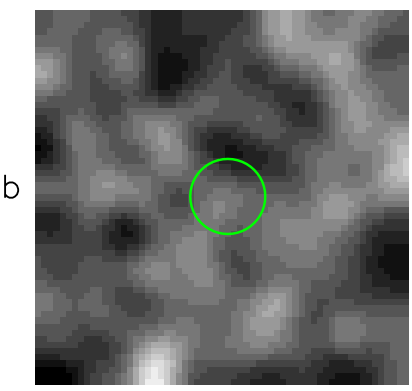

Hard Band

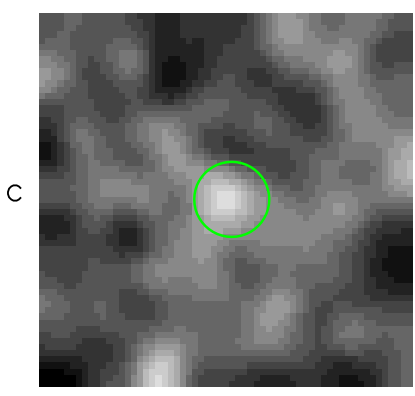

Hard Band
FIG. 1.- Weighted, stacked CDF-S 4 Ms data at the locations of the 151 $z=6$ galaxies in the sample of T11 smoothed by a 7 pixel $(3.5$ arcsec) Gaussian. Each image is 30 arcsec on a side and the green circle has radius 3 arcsec. The greyscale range is from median $-3 \sigma$ (black) to median $+5 \sigma$ (white). The left panels are for the soft band $(0.5-2 \mathrm{keV})$ and the right panels are for the hard band $(2-8 \mathrm{keV})$. The upper two panels show method (a) background subtraction, i.e. no $3 \sigma$ clipping. The middle two panels show method (b) where pixels within a radius of 22 arcsec of the target position have all pixels $>3 \sigma$ from the mean set to the mean value. The lower two panels show method (c) where only pixels in the background annulus (from $2 r$ to 22 $\operatorname{arcsec}$ ) are subject to $3 \sigma$ clipping. It is clear that in both bands, there is only evidence for a significant central stacked signal when only the background annulus is subject to $3 \sigma$ clipping.

Bouwens et al. (2006). They used method (a) stacking without any optimization for the off-axis dependent PSF. They considered two samples with different constraints on how close the LBGs can lie to a neighboring X-ray source in Xue et al. (2011). Their samples contained 210 (77) galaxies with no neighbor closer than 10 (22) arcsec. The stacked images for each sample in a range of X-ray bands show no significant positive signal at the center. Fiore et al. (2011b) quote $3 \sigma$ limits for the average count rate from the 210 galaxies of $<3.4 \times 10^{-7} \mathrm{ct} \mathrm{s}^{-1}$ and $<5.8 \times 10^{-7} \mathrm{ct} \mathrm{s}^{-1}$ in the soft and hard bands, respectively. The results presented here are consistent with, and a little more stringent than, those of Fiore et al., mainly due to the use of optimized apertures and weighting.

It is worth noting that the distribution of the $\mathrm{S} / \mathrm{N}$ of each target as a function of the weighting factor is a useful diagnostic for a true versus spurious signal. I have applied method (a) stacking to the samples of infrared star-formation rate excess (ISX) and infrared star-formation rate normal (ISN) galaxies
TABLE 1

STATISTICS OF Chandra CDF-S $z=6$ GALAXY STACKING

\begin{tabular}{ccc}
\hline $\begin{array}{c}\text { Background } \\
\text { method }\end{array}$ & $\begin{array}{c}\text { Soft band } \\
\text { S/N }\end{array}$ & $\begin{array}{c}\text { Hard band } \\
\text { S/N }\end{array}$ \\
\hline (a) & -1.3 & 0.1 \\
(b) & -0.1 & 1.1 \\
(c) & 4.7 & 6.1 \\
(c*) & 4.7 & 6.7 \\
\hline
\end{tabular}

NoteS.- Measured S/N of the stacked signal of the $151 z=6$ galaxy positions in T11 for the soft and hard bands using the three methods of background subtraction. ( $c^{*}$ ) gives the results for the $141 z=6$ galaxy positions which are further than 19 arcsec from an X-ray source in the 4 Ms catalog of Xue et al. (2011).

in the CDF-S stacked by Luo et al. (2011). Both samples show significant stacked signals because these are galaxies similar to those detected in the CDF-S, but with fluxes just below the detection threshold. These samples show a positive correlation between $\mathrm{S} / \mathrm{N}$ and weighting factor, because the sources have similar signal but those with lower weight have higher noise. In contrast, the $\mathrm{S} / \mathrm{N}$ against weighting factor using method (c) stacking of the $z=6$ LBG sample shows a negative correlation. This is because the lowest weight objects have larger photometry apertures $r$ and hence higher integrated backgrounds and a greater problem of insufficient background removal.

T11 required the criterion that the $z=6$ galaxies not have a neighboring X-ray source from the $2 \mathrm{Ms}$ catalog of Luo et al. (2008) within 22 arcsec. Since the 4 Ms catalog of Xue et al. (2011) is now available, its use to avoid nearby X-ray sources is preferable because it contains more sources. However, using a criterion of $22 \operatorname{arcsec}$ with the $4 \mathrm{Ms}$ catalog reduces the number of $z=6$ galaxies to stack to 110 . For the following analysis, the 22 arcsec criterion is relaxed to $19 \operatorname{arcsec}$ so a similar sample size of $141 \mathrm{LBGs}$ is retained. The outer limit of the background aperture is set to 18 arcsec to limit the influence of known sources just outside the aperture. As shown in the lowest row of Table 1, this minor change in sample and background determination does not significantly change the results compared to using method (c) with the 151 sources from T11.

A simple test of the robustness of any stacking method is to stack at random positions in the sky or at positions of sources known to not emit at the relevant waveband (e.g. White et al. 2007). The expected signal should be zero with a distribution about zero that gives the dispersion due to noise from the background. To quantify the size of the $\mathrm{S} / \mathrm{N}$ bias using method (c), a Monte-Carlo process of stacking the CDF-S Chandra maps at random locations is performed. To obtain a similar sample size and off-axis angle distribution to T11, the random locations are chosen to be offset from known $z=6$ galaxies (Bouwens et al. 2006) by distances randomly drawn in the range 15 to 30 arcsec in right ascension and declination. The same selection criteria of off-axis angle less than 9 arcmin and no neighbor X-ray source within 19 arcsec are applied. At the new random positions the same process of $\mathrm{S} / \mathrm{N}$ stacking was carried out using method (c) background subtraction.

This process was repeated using 500 sets of random locations and the resulting distribution of $\mathrm{S} / \mathrm{N}$ values are shown in Fig.2 The typical number of positions stacked was $144 \pm 8$. In every single one of the 500 trials there is a significant $(>3 \sigma)$ positive signal at random locations. The typical values for both the soft and hard bands are $\mathrm{S} / \mathrm{N}=6.5$. For com- 


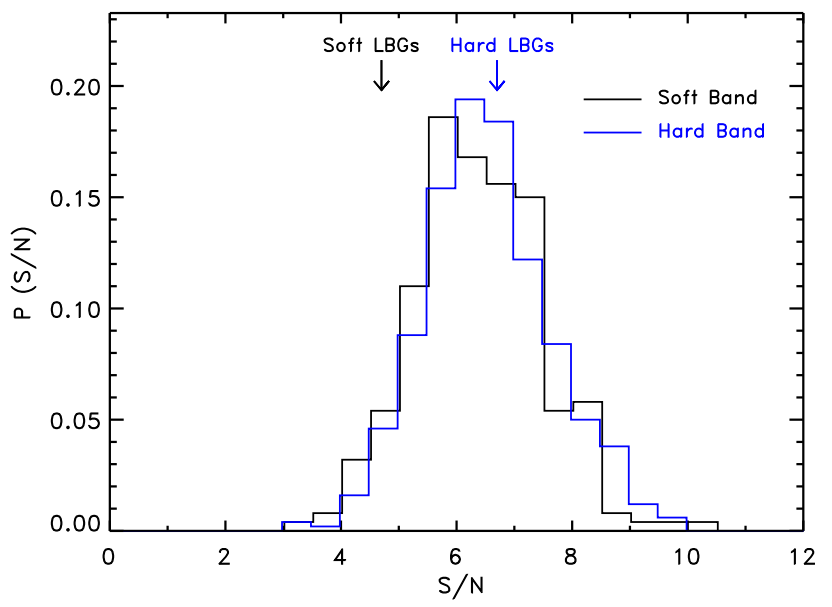

FIG. 2.- Probability distribution of measured S/N for stacks of $\approx 144$ random locations within the CDF-S using method (c) of $3 \sigma$ clipping only the background annulus pixels. The soft band results are shown in black and the hard band in blue. The measured $\mathrm{S} / \mathrm{N}$ of the stacks in each band at the true locations of the $z=6$ galaxies are shown by arrows. One can see that these are indistinguishable from the typical results obtained at random locations.

parison, the values found at the $z=6$ galaxy positions ( $\mathrm{c}^{*}$ in Table 1) are shown by arrows. For the hard band this is at approximately the peak of the distribution. For the soft band the observed S/N of 4.7 is at the lowest $10 \%$ of the distribution, so as before it is found that these target positions just happen to be located at mild flux deficits in the soft band images. It is concluded that method (c) background subtraction always leads to a false positive signal, due to under-estimation of the true background level.

\section{CONCLUSIONS}

It has been demonstrated that there is no evidence for a stacked X-ray signal in either the soft or hard bands at the positions of photometrically-selected $z=6$ galaxy candidates. The positive signal determined previously is due to an underestimation of the background due to $3 \sigma$ clipping of the background pixels. Stacking random locations within the X-ray images with this method leads to similar positive signals as found at the LBG positions. The hardness of the X-ray signal found by $\mathrm{T} 11$ is a combination of the fact that the $z=6$ galaxies happen to lie at locations with below average flux in the soft X-ray image and that the X-ray background is higher in the hard band than the soft band, with the latter effect dominant.

The conclusion is that there is no evidence yet at $z=6$ for either a very high black hole mass density or a sharp increase in the ratio of obscured to unobscured black holes. The determination of the $z=6$ black hole mass function by Willott et al. (2010) based on moderate and high luminosity optical quasars remains the most reliable estimate of this function, despite the considerable uncertainties due to quasar duty cycle and ratio of obscured to unobscured quasars. As noted by Willott et al. (2010) the $z=6$ black hole mass density is substantially lower than the $z=6$ stellar mass density expectation if the black hole - galaxy correlation does not evolve. The function is also flatter than expected based on models of Eddingtonlimited black hole growth. Volonteri \& Stark (2011) show that negative evolution of the black hole - galaxy mass correlation (or equivalently, a substantial fraction of high-redshift galaxies without black holes) and a steeper correlation than found locally, perhaps due to a correlation between accretion efficiency and halo mass, are required to fit the observed $z=6$ black hole mass function. Fiore et al. (2011a) suggest there is a mass-dependence to the active black hole duty cycle (similar to as observed at low-redshift) such that at high-redshift, most high-mass black holes are active, but most low-mass black holes are not. This would explain the flatter active black hole mass function and suggest there is a large, hidden population of high-redshift black holes, but that they are not rapidly growing.

Thanks to Ezequiel Treister for providing the data file for the CDF-S sources used in their analysis and for discussions regarding the differences between our stacking methods. Thanks also to Matt Jarvis for reading the manuscript and providing comments and to the anonymous referee for providing useful suggestions for improvement.

\section{REFERENCES}

Alexander, D. M., Bauer, F. E., Brandt, W. N., et al. 2003, AJ, 126, 539

Bouwens, R. J., Illingworth, G. D., Blakeslee, J. P. \& Franx, M. 2006, ApJ, 653,53

Bouwens, R. J., Illingworth, G. D., Franx, M., et al. 2009, ApJ, 705, 936

Ferrarese, L., \& Merritt, D. 2000, ApJ, 539, L9

Fiore, F., Puccetti, S., Grazian, A., et al. 2011a, A\&A, in press , arXiv: 1109.2888

Fiore, F., Puccetti, S., \& Mathur, S. 2011b, AdAst, submitted, arXiv:1109.4586

Gebhardt, K., Bender, R., Bower, G., et al. 2000, ApJ, 539, L13

Lauer, T. R., Tremaine, S., Richstone, D., \& Faber, S. M. 2007, ApJ, 670, 249

Luo, B., Bauer, F. E., Brandt, W. N., et al. 2008, ApJS, 179, 19
Luo, B., Brandt, W. N., Xue, Y. Q., et al. 2011, ApJ, 740, 37

Markevitch, M., Bautz, M. W., Biller, B., et al. 2003, ApJ, 583, 70

Stark, D., Ellis, R. S., Bunker, A., et al. 2009, ApJ, 697, 1493

Treister, E., Cardamone, C. N., Schawinski, K., et al. 2009, ApJ, 706, 535

Treister, E., Schawinski, K., Volonteri, M., Natarajan, P. \& Gawiser, E.

2011, Nature, 474, 356 (T11)

Volonteri, M., \& Stark, D. P. 2011, MNRAS, in press, arXiv:1107.1946

Wang, R., Carilli, C. L., Neri, R., et al. 2010, ApJ, 714, 699

White, R. L., Helfand, D. J., Becker, R. H., Glikman, E., \& de Vries, W.

2007, ApJ, 654, 99

Willott, C. J., Albert, L., Arzoumanian, D., et al. 2010, AJ, 140, 546

Xue, Y. Q., Luo, B., Brandt, W. N., et al. 2011, ApJSS, 195, 10 